\title{
Synthesis of Gold Nanoparticles Dispersed in Palm Oil Using Laser Ablation Technique
}

\author{
Amir Reza Sadrolhosseini, ${ }^{1}$ Suraya Abdul Rashid, ${ }^{1}$ and Azmi Zakaria ${ }^{2}$ \\ ${ }^{1}$ Materials Processing and Technology Laboratory, Nanomaterials and Nanotechnology Group, Institute of Advanced Technology, \\ Universiti Putra Malaysia (UPM), 43400 Serdang, Selangor, Malaysia \\ ${ }^{2}$ Department of Physics, Faculty of Science, Universiti Putra Malaysia (UPM), 43400 Serdang, Selangor, Malaysia
}

Correspondence should be addressed to Suraya Abdul Rashid; suraya_ar@upm.edu.my

Received 6 December 2016; Revised 13 February 2017; Accepted 21 February 2017; Published 5 April 2017

Academic Editor: Jorge Pérez-Juste

Copyright (c) 2017 Amir Reza Sadrolhosseini et al. This is an open access article distributed under the Creative Commons Attribution License, which permits unrestricted use, distribution, and reproduction in any medium, provided the original work is properly cited.

Gold nanoparticles have more applications in biology, medicine, and industry. In this study, gold nanoparticles were synthesized in pure palm oil using laser ablation technique. Gold nanoparticles were fabricated in different temperature, and the effect of the temperature on the particle size was investigated. Consequently, the tail of the carbonyl band of fatty acids was capped gold nanoparticles, and spherically shaped gold nanoparticles with size range of 8.92 to $19.73 \mathrm{~nm}$ were formed in palm oil. The temperature caused the agglomeration of nanoparticles while the particle size increased with an increase in the temperature.

\section{Introduction}

The application of gold nanoparticles (Au-NPs) in drug delivery, microelectronics, environments, photonics, electronics [1], photodynamic therapy [2], therapeutic agent delivery [3], biosensors, sensors [4], medical diagnoses [5], and catalysis have been considered by many researchers. Gold nanoparticles have particular chemical and physical properties related to shape, size $[6,7]$, and surrounding medium of NPs $[6,8]$. Numerous factors influence the shape and size of nanoparticles and one of each significant factor is temperature [9].

There was a strong interaction between electrical field of light and gold nanoparticles [10] while the surface plasmon absorption occurred in visible range [11]. The coherent excitation of free electrons causes the surface plasmon band in colloidal Au-NPs [12]. When the light beam interacts with Au-NPs, the response of Au-NPs strongly depends on particle size and concentration of Au-NPs in the liquid [13]. Many methods were presented to prepare Au-NPs including chemical methods $[14,15]$, microwave method [16], and sonochemical method [17]. Au-NPs were prepared in dimethyl sulfoxide, acetonitrile, tetrahydrofuran $[18,19]$, and nonorganic liquid [19] using laser ablation.
The main problem in the fabrication of $\mathrm{Au}-\mathrm{NPs}$ is collapsing and agglomeration of Au-NPs in liquid due to thermodynamic principals. This challenge can be harnessed by improving the surrounding medium which prevents $\mathrm{Au}$ NPs agglomeration through charge and steric stabilization. Hence, many researchers have made efforts to improve the stabilizers $[20,21]$ in order to counter the agglomeration of NPs in aqueous solution.

Recently, some fatty acids or vegetable oils were used as stabilizers [22, 23]. Fatty acids have amphiphilic molecules and they have a polar carboxylic group, which can cap AuNPs. Consequently, nonpolar long carbon chains can prevent Au-NPs agglomeration through steric repulsion.

Past research has reported the preparation of Au-NPs in vegetable oils as stabilizing agents [24]. The best commercial vegetable oil traded in the world market is palm oil which contains palmitic $44.3 \%$, stearic $4.6 \%$, myristic $1 \%$, oleic $38.7 \%$, and linoleic $10.5 \%$ (Figure 1). It is cheaper and very stable for oxidation than canola, soybean, and rapeseed oil [25]. Palm oil contains the presence of long hydrocarbon chains and polar ester bond which makes it a good choice for stabilizing NPs (Figure 1). 


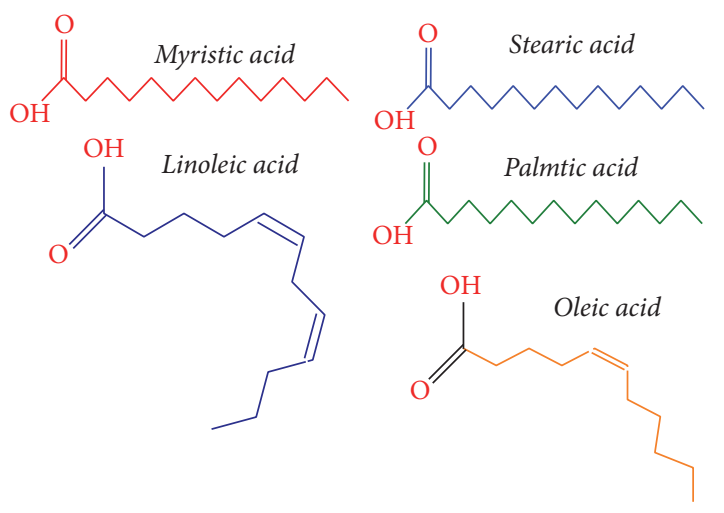

FIGURE 1: The chemical structure of fatty acids in palm oil contains stearic acid, palmitic acid, myristic acid, oleic acid, and linoleic acid.

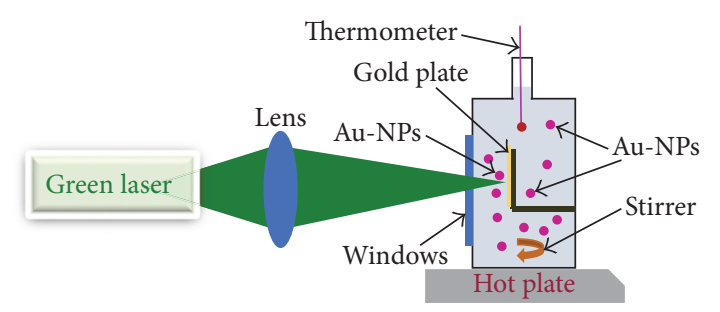

FIgURE 2: Laser ablation setup for fabrication of Au-NPs in palm oil contains a Nd:YAG laser, a lens, a gold plate, a hotplate, a thermometer, and a stirrer.

In this research, Au-NPs were synthesized in pure palm oil using laser ablation method in different temperature. The capping of the nanoparticles, particle size, and particle shape were investigated using Fourier transfer infrared spectroscopy, UV-visible spectroscopy, and transmission electron microscopy to test the stabilizing ability of palm oil.

\section{Methods}

2.1. Laser Ablation Synthesis of Gold Nanoparticle. In this experiment, a gold plate (Aldrich, high impurity 99.99\%) was immersed in $10 \mathrm{~mL}$ of pure palm oil. A laser beam of $532 \mathrm{~nm}$ wavelength with duration of $10 \mathrm{~ns}$ and $1200 \mathrm{~mJ}$ ablated the gold plate in different temperature for 10 mins with $30 \mathrm{~Hz}$ repetition rate. Figure 2 shows the laser ablation setup which contains a Q-switched Nd:YAG laser, a solution container, a gold plate, a lens $(f=30 \mathrm{~cm})$, a hot plate with stirrer, and a thermometer. The solution was heated using the hot plate, and the temperature of the solution was measured using the thermometer.

Gold nanoparticles formed in pure palm oil during laser ablation of the gold plate with ablation time of 20 minutes. The experiment was continued in different temperatures as $40^{\circ} \mathrm{C}, 64^{\circ} \mathrm{C}, 75^{\circ} \mathrm{C}, 83^{\circ} \mathrm{C}$, and $92^{\circ} \mathrm{C}$. In order to make sure that gold nanoparticles were dispersed evenly in palm oil, the solution was stirred during the ablation of the gold plate, and the solution container was moved horizontally to obtain the fresh surface area.

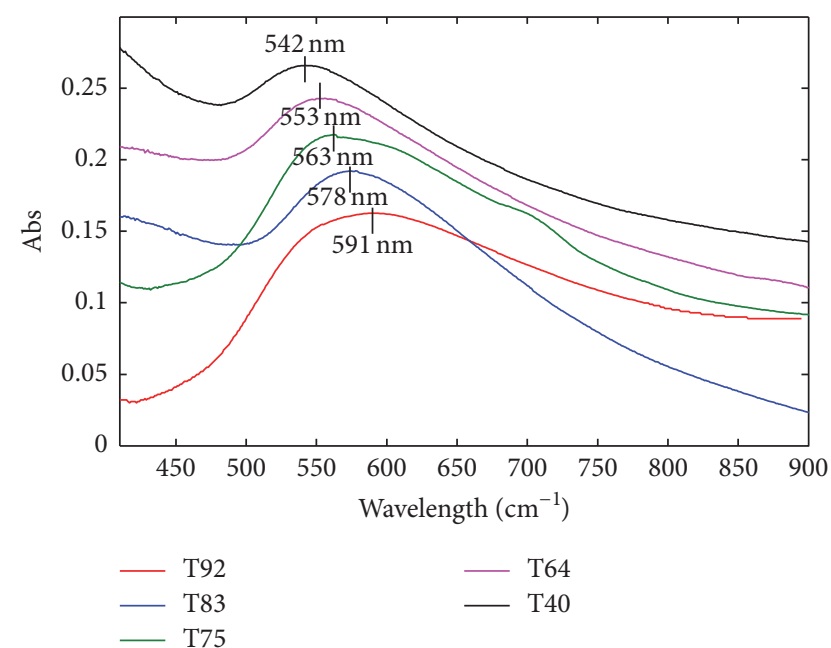

FIgURE 3: UV-visible results show the intensity peak having a red shift with increasing the temperature.

The UV-visible spectrum, morphology, size, and concentration of the prepared samples were obtained using the UVvisible double-beam spectrophotometer (Shimadzu), transmission electron microscopy (TEM, Hitachi H-7100, Hitachi, Chula Vista, CA), Fourier transform infrared spectroscopy (FT-IR), and atomic absorption spectroscopy (AAS, S series), respectively.

\section{Results and Discussion}

Figure 3 shows the UV-visible spectrum of palm oil with gold nanoparticles and the peaks appeared at $542 \mathrm{~nm}, 553 \mathrm{~nm}$, $563 \mathrm{~nm}, 578$, and $591 \mathrm{~nm}$. These peaks arose from a localized surface plasmon resonance of Au-NPs. Hence, they confirmed that $\mathrm{Au}-\mathrm{NPs}$ were formed inside palm oil. The ablation time was 20 minutes while the temperatures of palm oil during the ablation of the gold target were $40^{\circ} \mathrm{C}, 64^{\circ} \mathrm{C}, 75^{\circ} \mathrm{C}$, $83^{\circ} \mathrm{C}$, and $92^{\circ} \mathrm{C}$. The peak intensity of UV-visible spectrum decreased with an increase in the temperature, and the red shift is observed in the UV-visible spectrum. In accordance with Mie theory, the red shift authenticates the increase in particle size. Consequently, the particle size increases with an increase in the temperature.

The concentrations of Au-NPs measured using atomic absorption spectroscopy for the sample at temperatures of $40^{\circ} \mathrm{C}, 64^{\circ} \mathrm{C}, 75^{\circ} \mathrm{C}, 83^{\circ} \mathrm{C}$, and $92^{\circ} \mathrm{C}$ were $21.3,13.4,10.8,9.1$, and $8.6 \mathrm{mg} / \mathrm{L}$, respectively. The volume fraction of each sample was obtained using the following equation $[26,27]$ :

$$
V=\frac{V_{p}}{V_{p}+V_{L}}
$$

where $V_{L}$ and $V_{p}$ are the volume of palm oil and the volume of Au-NPs ( $m / \rho$, where $\rho$ and $m$ are the mass density of gold and the mass of the particles dispersed in palm oil, resp.). Hence, 


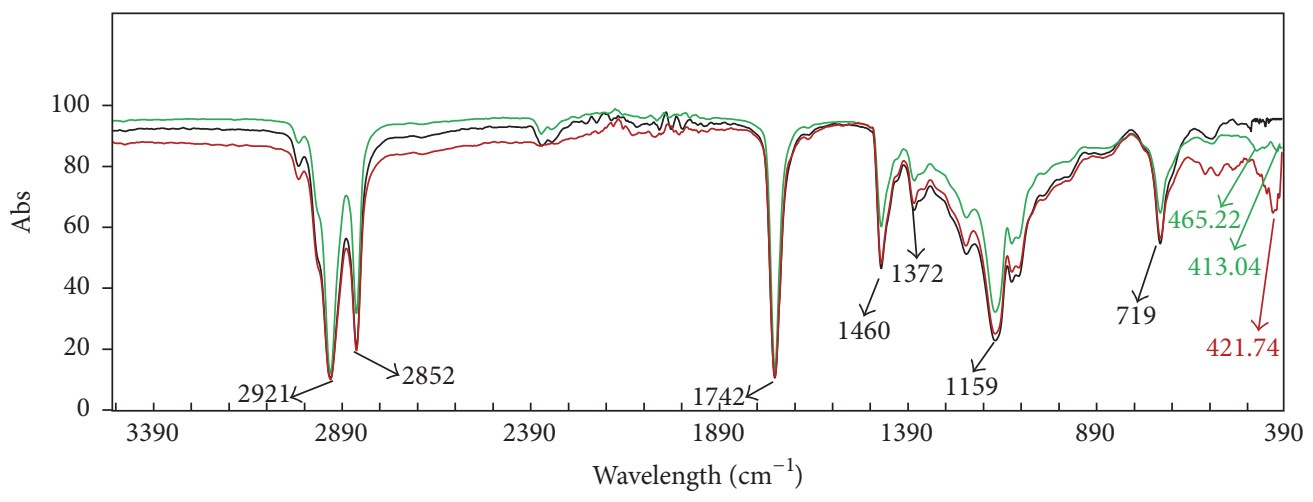

(a) Pure palm oil
(b) Au-NPs T40
(c) Au-NPs T92

FIgURE 4: FT-IR results related to pure palm oil and palm oil with Au-NPs in $40^{\circ} \mathrm{C}$ and $90^{\circ} \mathrm{C}$.

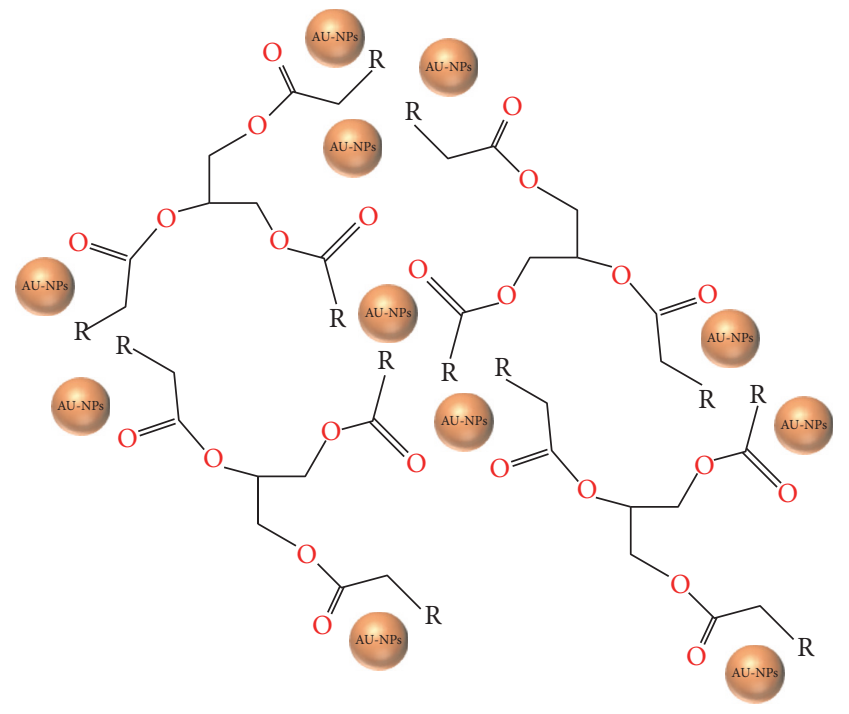

FIGURE 5: Au-NPs capped with fatty acids in palm oil.

(2) was derived from modification and a simplification of (1) as follows:

$$
V=\frac{C_{\text {Particle }}}{C_{\text {Particle }}+\rho},
$$

where $C_{\text {Particle }}$ are the concentrations of Au-NPs obtained from the AAS. The volume fraction of Au-NPs was obtained in the range of $0.1104 \times 10^{-5}$ to $0.0446 \times 10^{-5}$ which decreased with an increase in the temperature of the sample (see Table 1).

Figure 4 shows the FT-IR spectrum analysis of pure palm oil and palm oil/Au-NPs. The spectra wavelength ranges from $400 \mathrm{~cm}^{-1}$ to $3400 \mathrm{~cm}^{-1}$, and they show a chemical structure of palm oil and palm oil/Au-NPs. Since the effect of temperature has been investigated for the preparation of Au-NPs, the FT-IR results related to nanofluid which was formed in $40^{\circ} \mathrm{C}$ (Au-NPs T40) and $92^{\circ} \mathrm{C}$ (Au-NPs T92) were presented.
TABLE 1: The pertinent parameters of palm oil/Au-NPs nanofluid.

\begin{tabular}{lcccc}
\hline Gold-NPs & $\begin{array}{c}\text { Temperature } \\
\left({ }^{\circ} \mathrm{C}\right)\end{array}$ & $\begin{array}{c}\text { Concentration } \\
(\mathrm{mg} / \mathrm{L})\end{array}$ & $\begin{array}{c}\text { Particle size } \\
(\mathrm{nm})\end{array}$ & $\begin{array}{c}\text { Volume } \\
\text { fraction } \\
\left(\times 10^{-5}\right)\end{array}$ \\
\hline Au-NPs T40 & 40 & 21.3 & 8.92 & 0.1104 \\
Au-NPs T64 & 64 & 13.4 & 9.80 & 0.0694 \\
Au-NPs T75 & 75 & 10.8 & 12.87 & 0.0560 \\
Au-NPs T83 & 83 & 9.1 & 15.07 & 0.0472 \\
Au-NPs T92 & 92 & 8.6 & 19.73 & 0.0446 \\
\hline
\end{tabular}

Figure 4(a) shows the FT-IR spectrum of pure palm oil where the peaks at 2921, 2852, and $1742 \mathrm{~cm}^{-1}$ correspond with the $=\mathrm{C}-\mathrm{H}$ stretching vibration, $\mathrm{CH}_{2}$ asymmetric and symmetric bending, and $-\mathrm{C}=\mathrm{C}$ stretching ester carbonyl function group of triglycerides, respectively.

In addition, the peaks of $1460,1372,1159$, and $719 \mathrm{~cm}^{-1}$ are related to the $\mathrm{CH}_{2}$ bending, -C-O stretching vibration, and $-\mathrm{CH}_{2}$ bending (cis $-\mathrm{CH}=\mathrm{CH}$ - bending) $[28,29]$. Figures 4(b) and 4(c) depict the FT-IR spectrum of samples after the ablation of the gold target in palm oil. The peaks at 2921, 1742, $1460,1372,1159$, and $719 \mathrm{~cm}^{-1}$ are related to pure palm oil and the peaks in $413.04,421.74$, and $465.22 \mathrm{~cm}^{-1}$ are related to $\mathrm{Au}-\mathrm{NPs}[28,30]$ that formed inside palm oil using laser ablation of the gold plate. The peaks at 2921, 1742, 1460, 1372,1159 , and $719 \mathrm{~cm}^{-1}$ remain stable during synthesis of Au-NPs in palm oil while other peaks related to formation of another component such as graphitic compound did not appear. Therefore, there was no mutation of the molecular structure and functional groups of palm oil during laser ablation of the gold plate.

As a result, palm oil components can be used as capping agents for Au-NPs. Figure 5 shows the model of capping the nanoparticles. The tail of the carbonyl band capped Au-NPs through the electron transfer from the carboxylic group to $\mathrm{Au}-\mathrm{NPs}$ thereby causing an impediment of the motion of the molecules inside the particular area with a decrease in 


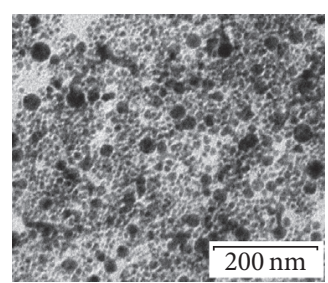

(a1)

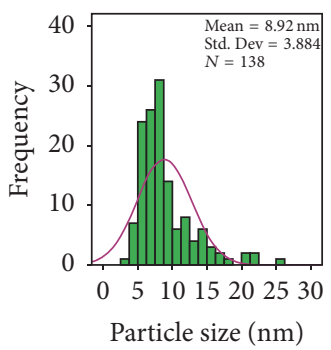

(a2)

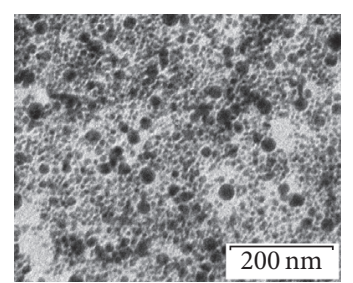

(b1)

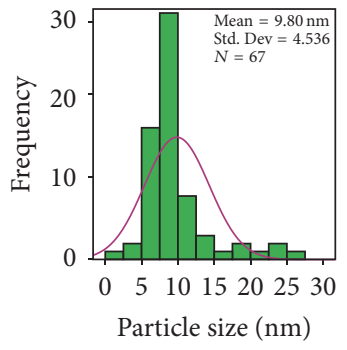

(b2)

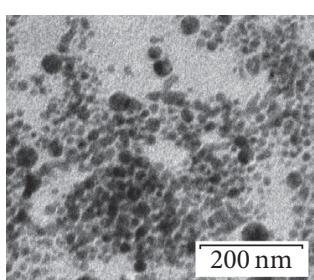

(c1)

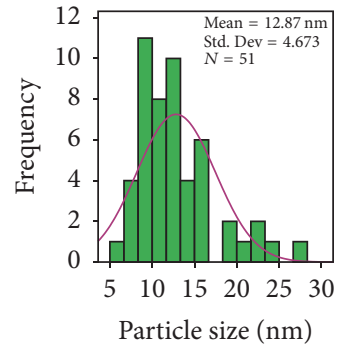

(c2)

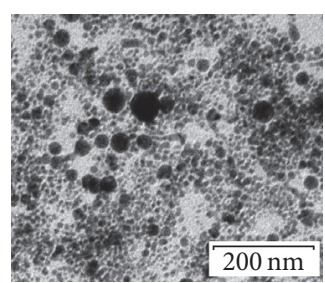

(d1)

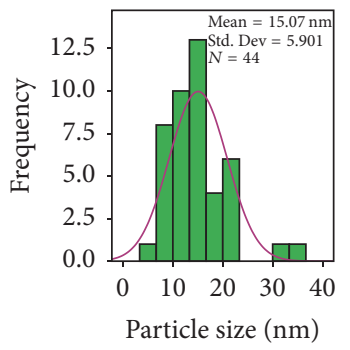

$(\mathrm{d} 2)$

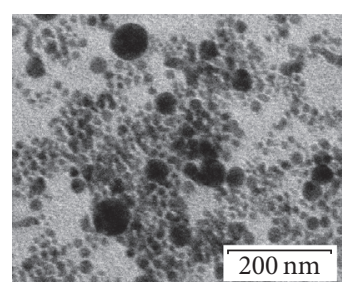

$(\mathrm{e} 1)$

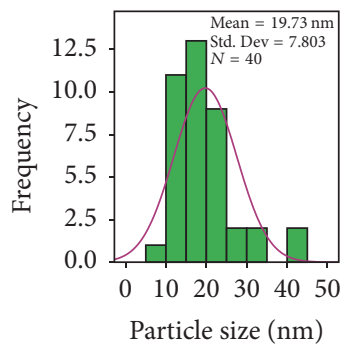

(e2)

FIgURE 6: The TEM images demonstrate the Au-NPs formed in palm oil in spherical shape.

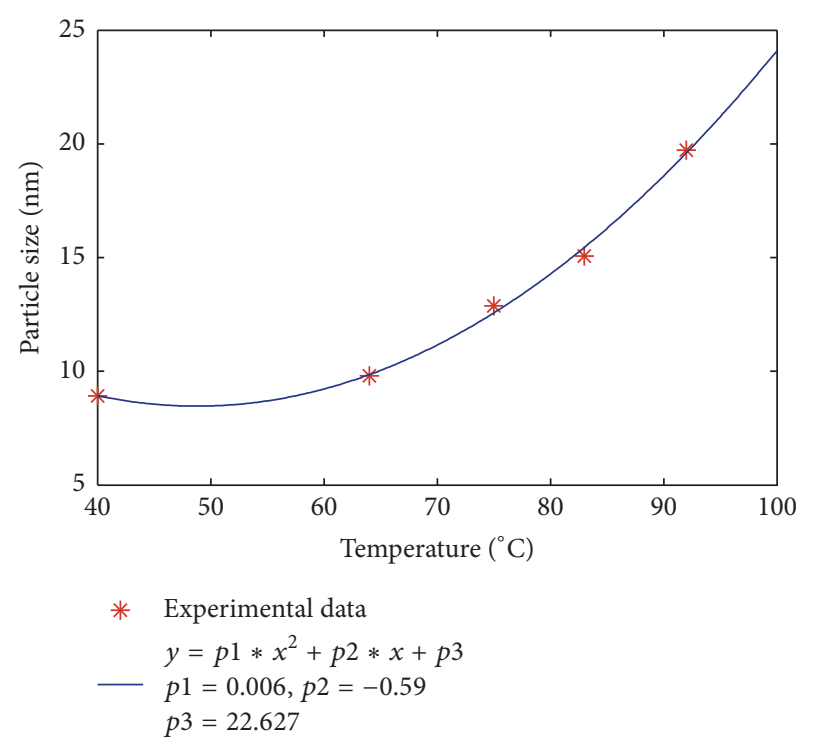

FIGURE 7: Variation of particle size with temperature.

entropy $[28,30]$. This proves that a long hydrocarbon chain prevents the agglomeration of Au-NPs.

Figures 6(a1), 6(a2), 6(b1), 6(b2), 6(c1), 6(c2), 6(d1), 6(d2), 6(e1), and 6(e2) show the TEM images and their analysis. It was observed that Au-NPs formed in the spherical form. The particle size was achieved after analyzing the TEM image using the UTHSCSA image tool software program. As a result, the particle size was in the range of 8.92 to $19.73 \mathrm{~nm}$. Figure 7 shows the variation of particle size according to the temperature. The pertinent parameters were sorted in Table 1.

Temperature of a sample is a significant parameter for formation of the nanoparticles during ablation of the gold target. The effect of the temperature appeared in the size of the nanoparticles, and the final size was achieved due to agglomeration of Au-NPs inside palm oil. At initial step, laser beam causes the phase transition and nucleation of Au-NPs near the target inside palm oil. Consequently, Au-NPs cluster was formed in spherical shape and small size, and Au-NPs were dispersed in palm oil. When the temperature of palm oil was increased, in accordance with Maxwell-Boltzmann kinetic energy [9, 31], the probability of agglomeration of $\mathrm{Au}$ NPs cluster increased therefore causing an increase in particle size as the temperature increased.

\section{Conclusions}

Gold nanoparticles formed in palm oil in the spherical shape with particle size ranging from 8.92 to $19.73 \mathrm{~nm}$. The heat had an effect on the particle size thereby causing an increase in the particle size as the temperature increased. The localized surface plasmon resonance peaks appeared in the green range of UV-visible spectrum. The tail of carbonyl band capped AuNPs. Consequently, the laser ablation technique is a green and simple method for the synthesis of Au-NPs in the presence of fatty acids.

\section{Conflicts of Interest}

The authors declare that they have no conflicts of interest.

\section{References}

[1] A. Tomar and G. Garg, "Short review on application of gold nanoparticles," Global Journal of Pharmacology, vol. 7, no. 1, pp. 34-38, 2013.

[2] M. K. K. Oo, Y. Yang, Y. Hu, M. Gomez, H. Du, and H. Wang, "Gold nanoparticle-enhanced and size-dependent generation of reactive oxygen species from protoporphyrin IX," ACS Nano, vol. 6, no. 3, pp. 1939-1947, 2012.

[3] L. Vigderman and E. R. Zubarev, "Therapeutic platforms based on gold nanoparticles and their covalent conjugates with drug 
molecules," Advanced Drug Delivery Reviews, vol. 65, no. 5, pp. 663-676, 2013.

[4] K. Saha, S. S. Agasti, C. Kim, X. Li, and V. M. Rotello, "Gold nanoparticles in chemical and biological sensing," Chemical Reviews, vol. 112, no. 5, pp. 2739-2779, 2012.

[5] W. Cai, T. Gao, H. Hong, and J. Sun, "Applications of gold nanoparticles in cancer nanotechnology," Nanotechnology, Science and Applications, vol. 1, pp. 17-32, 2008.

[6] J. J. Diao, J. Sun, J. B. Hutchison, and M. E. Reeves, "Self assembled nanoparticle wires by discontinuous vertical colloidal deposition," Applied Physics Letters, vol. 87, no. 10, pp. 103113-103116, 2005.

[7] V. M. Rentería and J. García-Macedo, "Modeling of optical absorption of silver prolate nanoparticles embedded in sol-gel glasses," Materials Chemistry and Physics, vol. 91, no. 1, pp. 8893, 2005.

[8] N. V. Tarasenko, A. V. Butsen, and E. A. Nevar, "Laser-induced modification of metal nanoparticles formed by laser ablation technique in liquids," Applied Surface Science, vol. 247, no. 1-4, pp. 418-422, 2005.

[9] S. L. Fiedler, S. Izvekov, and A. Violi, "The effect of temperature on nanoparticle clustering," Carbon, vol. 45, no. 9, pp. 17861794, 2007.

[10] N. J. Halas, S. Lal, W.-S. Chang, S. Link, and P. Nordlander, "Plasmons in strongly coupled metallic nanostructures," Chemical Reviews, vol. 111, no. 6, pp. 3913-3961, 2011.

[11] P. K. Jain, K. S. Lee, I. H. El-Sayed, and M. A. El-Sayed, "Calculated absorption and scattering properties of gold nanoparticles of different size, shape, and composition: applications in biological imaging and biomedicine," Journal of Physical Chemistry B, vol. 110, no. 14, pp. 7238-7248, 2006.

[12] A. C. Templeton, J. J. Pietron, R. W. Murray, and P. Mulvaney, "Solvent refractive index and core charge influences on the surface plasmon absorbance of alkanethiolate monolayerprotected gold clusters," Journal of Physical Chemistry B, vol. 104, no. 3, pp. 564-570, 2000.

[13] O. A. Yeshchenko, I. M. Dmitruk, A. A. Alexeenko, A. V. Kotko, J. Verdal, and A. O. Pinchuk, "Size and temperature effects on the surface plasmon resonance in silver nanoparticles," Plasmonics, vol. 7, no. 4, pp. 685-694, 2012.

[14] J. Turkevich, P. C. Stevenson, and J. Hillier, "A study of the nucleation and growth processes in the synthesis of colloidal gold," Discussions of the Faraday Society, vol. 11, pp. 55-75, 1951.

[15] Y. Li, T.-Y. Wu, S.-M. Chen, M. A. Ali, and F. M. A. AlHemaid, "Green synthesis and electrochemical characterizations of gold nanoparticles using leaf extract of Magnolia kobus," International Journal of Electrochemical Science, vol. 7, no. 12, pp. 1274212751, 2012.

[16] R. K. Das, P. J. Babu, N. Gogoi, P. Sharma, and U. Bora, "Microwave-mediated rapid synthesis of gold nanoparticles Using Calotropis procera latex and study of optical properties," ISRN Nanomaterials, vol. 2012, Article ID 650759, 6 pages, 2012.

[17] K. Okitsu, M. Ashokkumar, and F. Grieser, "Sonochemical synthesis of gold nanoparticles: effects of ultrasound frequency," The Journal of Physical Chemistry B, vol. 109, no. 44, pp. 2067320675, 2005.

[18] V. Amendola, S. Polizzi, and M. Meneghetti, "Laser ablation synthesis of gold nanoparticles in organic solvents," Journal of Physical Chemistry B, vol. 110, no. 14, pp. 7232-7237, 2006.

[19] E. V. Barmina, G. A. Shafeev, P. G. Kuzmin, A. A. Serkov, A. V. Simakin, and N. N. Melnik, "Laser-assisted generation of gold nanoparticles and nanostructures in liquid and their plasmonic luminescence," Applied Physics A: Materials Science and Processing, vol. 115, no. 3, pp. 747-752, 2014.

[20] A. Roucoux, J. Schulz, and H. Patin, "Reduced transition metal colloids: a novel family of reusable catalysts?" Chemical Reviews, vol. 102, no. 10, pp. 3757-3778, 2002.

[21] S. I. Stoeva, A. B. Smetana, C. M. Sorensen, and K. J. Klabunde, "Gram-scale synthesis of aqueous gold colloids stabilized by various ligands," Journal of Colloid and Interface Science, vol. 309, no. 1, pp. 94-98, 2007.

[22] N. Wu, L. Fu, M. Su, M. Aslam, K. C. Wong, and V. P. Dravid, "Interaction of fatty acid monolayers with cobalt nanoparticles," Nano Letters, vol. 4, no. 2, pp. 383-386, 2004.

[23] H.-T. Song, J.-S. Choi, Y.-M. Huh et al., "Surface modulation of magnetic nanocrystals in the development of highly efficient magnetic resonance probes for intracellular labeling," Journal of the American Chemical Society, vol. 127, no. 28, pp. 9992-9993, 2005.

[24] E. C. Da Silva, M. G. A. Da Silva, S. M. P. Meneghetti et al., "Synthesis of colloids based on gold nanoparticles dispersed in castor oil," Journal of Nanoparticle Research, vol. 10, no. 1, pp. 201-208, 2008.

[25] X. Bo, X. Guomin, C. Lingfeng, W. Ruiping, and G. Lijing, "Transesterification of palm oil with methanol to biodiesel over a $\mathrm{KF} / \mathrm{Al}_{2} \mathrm{O}_{3}$ heterogeneous base catalyst," Energy and Fuels, vol. 21, no. 6, pp. 3109-3112, 2007.

[26] R. Zamiri, A. Zakaria, H. A. Ahangar, A. R. Sadrolhosseini, and M. A. Mahdi, "Fabrication of silver nanoparticles dispersed in palm oil using laser ablation," International Journal of Molecular Sciences, vol. 11, no. 11, pp. 4764-4770, 2010.

[27] A. R. Sadrolhosseini, S. A. Rashid, A. S. M. Noor, A. Kharazmi, and L. A. Mehdipour, "Fabrication of silver nanoparticles in pomegranate seed oil with thermal properties by laser ablation technique," Digest Journal of Nanomaterials and Biostructures, vol. 10, no. 3, pp. 1009-1018, 2015.

[28] A. Rohman, T. Kuwat, S. Retno, Sismindari, E. Yuny, and W. Tridjoko, "Fourier transform infrared spectroscopy applied for rapid analysis of lard in palm oil," International Food Research Journal, vol. 19, no. 3, pp. 1161-1165, 2012.

[29] A. Rohman, Y. B. Che Man, A. Ismail, and P. Hashim, "Application of FTIR spectroscopy for the determination of virgin coconut oil in binary mixtures with olive oil and palm oil," Journal of the American Oil Chemists' Society, vol. 87, no. 6, pp. 601-606, 2010.

[30] A. R. Sadrolhosseini, A. S. M. Noor, N. Faraji, A. Kharazmi, and M. A. Mahdi, "Optical nonlinear refractive index of laserablated gold nanoparticles graphene oxide composite," Journal of Nanomaterials, vol. 2014, Article ID 962917, 8 pages, 2014.

[31] P. Minutolo, G. Gambi, A. D’Alessio, and S. Carlucci, "Spectroscopic characterisation of carbonaceous nanoparticles in premixed flames," Atmospheric Environment, vol. 33, no. 17, pp. 2725-2732, 1999. 

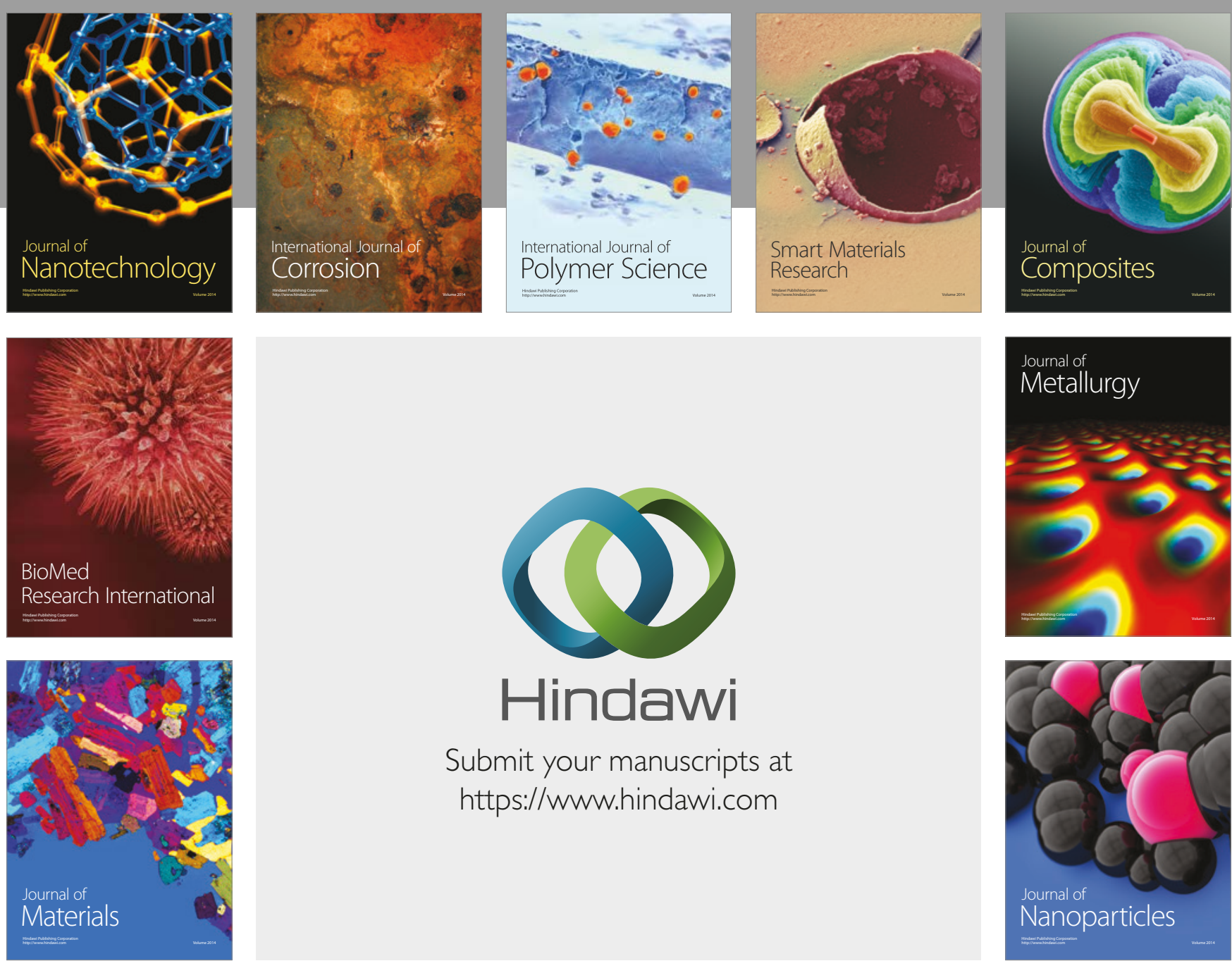

\section{Hindawi}

Submit your manuscripts at

https://www.hindawi.com
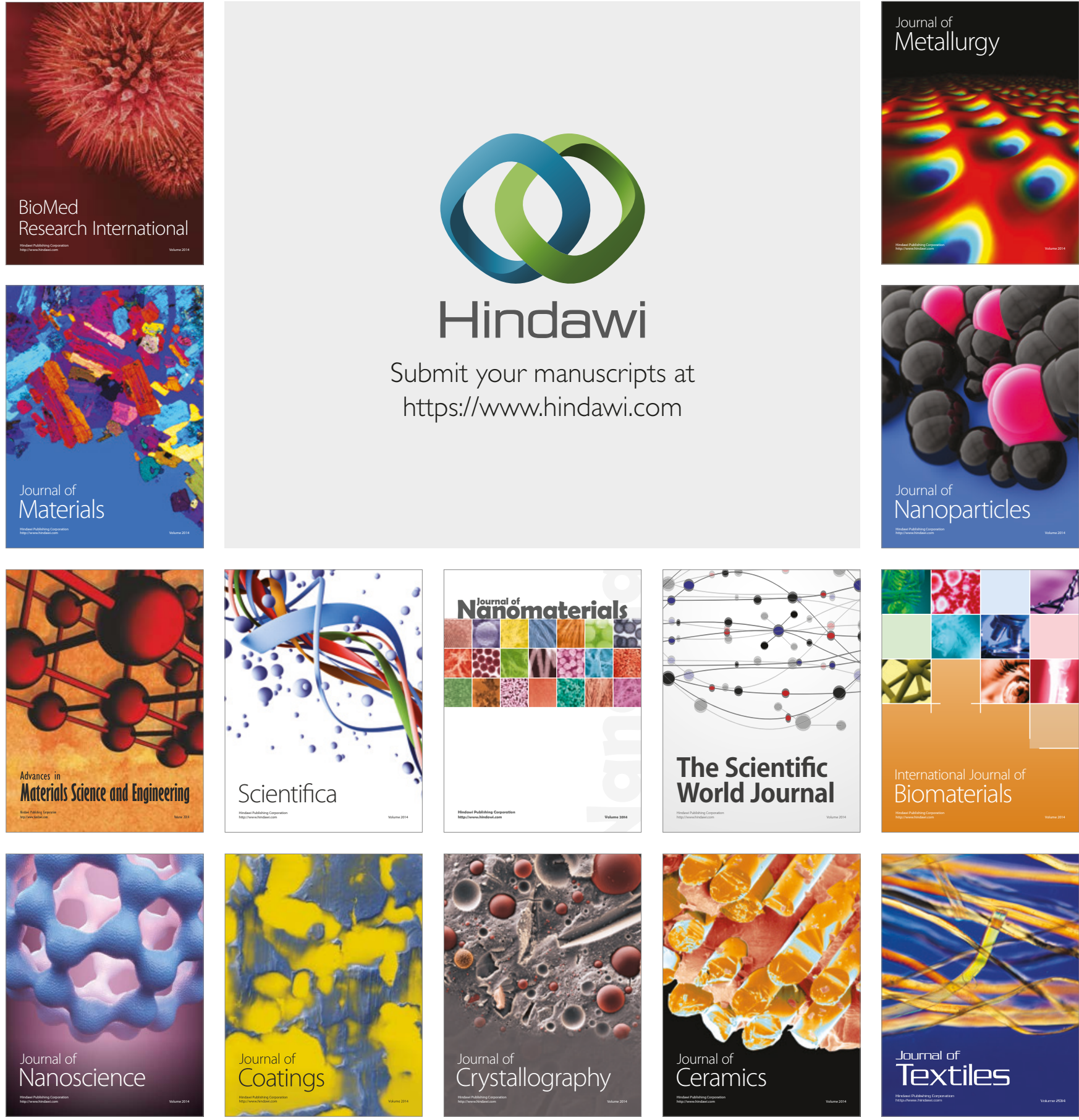

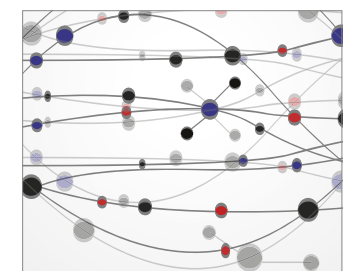

The Scientific World Journal
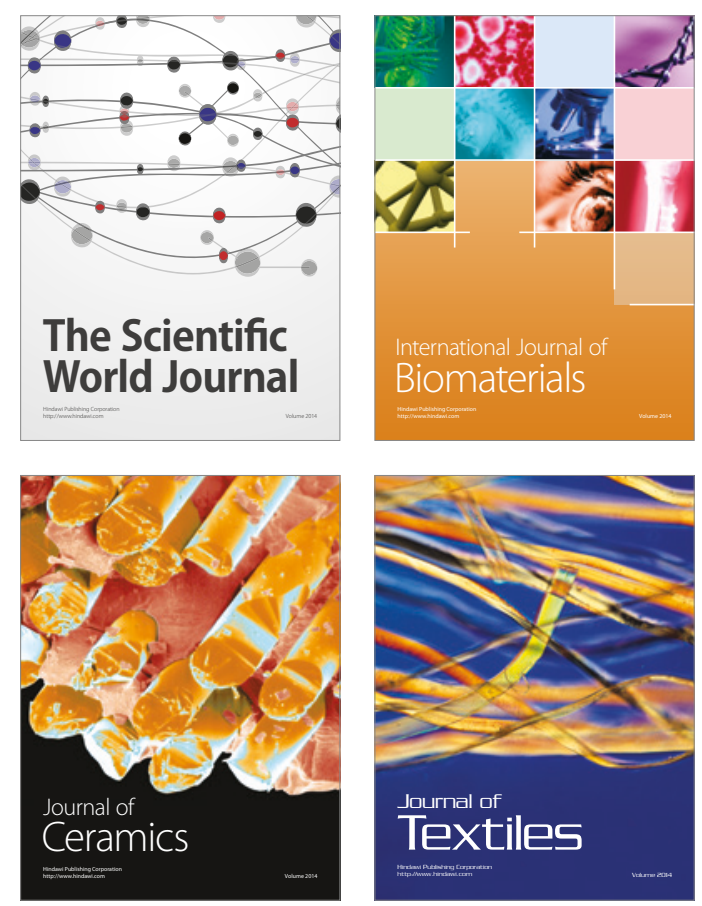